TITLE:

\title{
Mixed quantal-semiquantal dynamics with stochastic particles for backreaction.
}

AUTHOR(S):

Ando, Koji

\section{CITATION:}

Ando, Koji. Mixed quantal-semiquantal dynamics with stochastic particles for backreaction.. The Journal of chemical physics 2014, 141(14): 144106.

ISSUE DATE:

2014-10-14

URL:

http://hdl.handle.net/2433/191227

\section{RIGHT:}

Copyright 2014 American Institute of Physics. This article may be downloaded for personal use only. Any other use requires prior permission of the author and the American Institute of Physics 


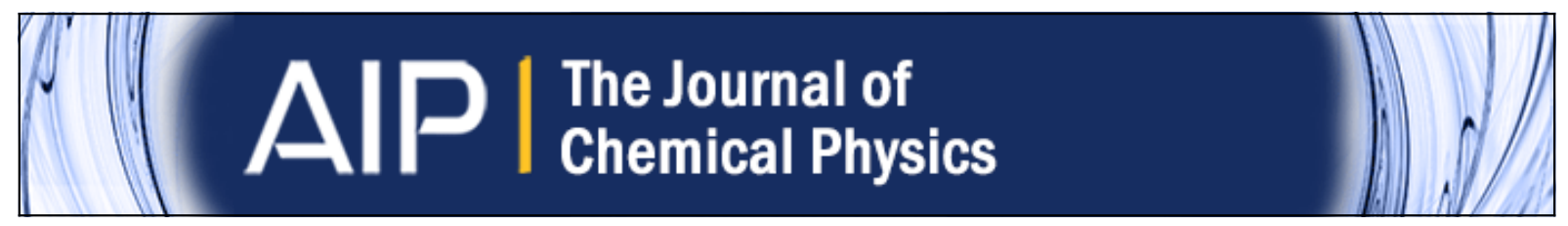

Mixed quantal-semiquantal dynamics with stochastic particles for backreaction

Koji Ando

Citation: The Journal of Chemical Physics 141, 144106 (2014); doi: 10.1063/1.4897532

View online: http://dx.doi.org/10.1063/1.4897532

View Table of Contents: http://scitation.aip.org/content/aip/journal/jcp/141/14?ver=pdfcov

Published by the AIP Publishing

\section{Articles you may be interested in}

Stochastic dynamics of complexation reaction in the limit of small numbers

J. Chem. Phys. 134, 195101 (2011); 10.1063/1.3590918

Stochastic deformation of integrable dynamical systems and random time symmetry

J. Math. Phys. 51, 082104 (2010); 10.1063/1.3467214

Fluctuating dynamics of nematic liquid crystals using the stochastic method of lines

J. Chem. Phys. 133, 044112 (2010); 10.1063/1.3455206

Mean-field dynamics with stochastic decoherence (MF-SD): A new algorithm for nonadiabatic mixed quantum/classical molecular-dynamics simulations with nuclear-induced decoherence

J. Chem. Phys. 123, 234106 (2005); 10.1063/1.2131056

Geometrical stochastic control and quantization

J. Math. Phys. 38, 173 (1997); 10.1063/1.531848

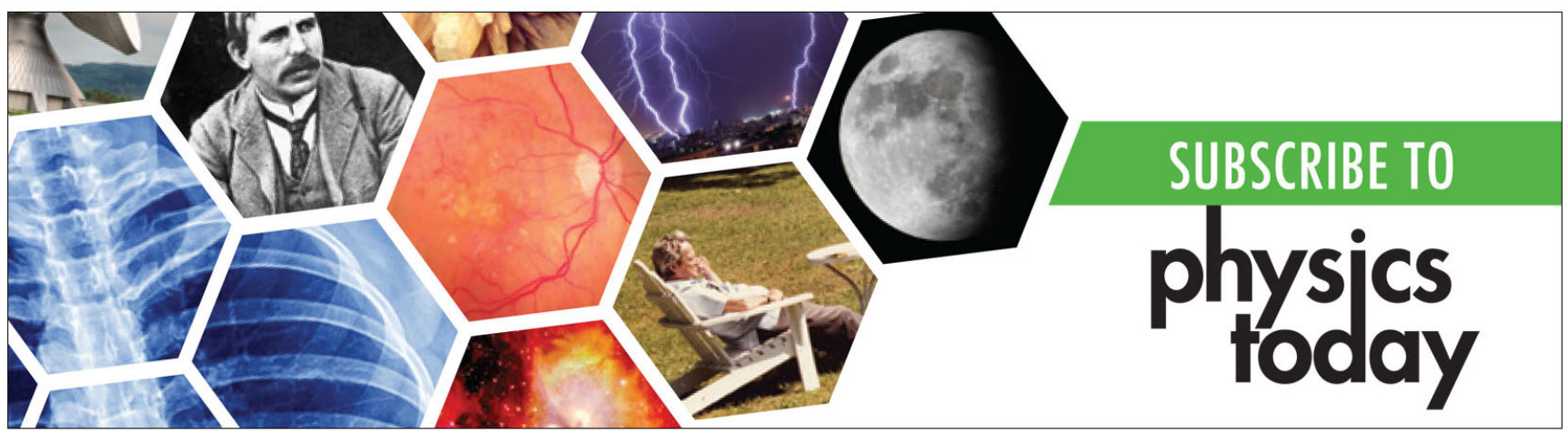




\title{
Mixed quantal-semiquantal dynamics with stochastic particles for backreaction
}

\author{
Koji Ando a) \\ Department of Chemistry, Graduate School of Science, Kyoto University, Sakyo-ku, Kyoto 606-8502, Japan
}

(Received 30 July 2014; accepted 29 September 2014; published online 14 October 2014)

\begin{abstract}
A mixed quantal-semiquantal theory is presented in which the semiquantal squeezed-state wave packet describes the heavy degrees of freedom. Starting from the mean-field equations of motion that are naturally derived from the time-dependent variational principle, we introduce the stochastic particle description for both the quantal and semiquantal parts in an aim to take into account the interparticle correlation, in particular the "quantum backreaction" beyond the mean-field approximation. A numerical application on a model of $\mathrm{O}_{2}$ scattering from a Pt surface demonstrates that the proposed scheme gives correct asymptotic behavior of the scattering probability, with improvement over the mixed quantum-classical scheme with Bohmian particles, which is comprehended by comparing the Bohmian and the stochastic trajectories. () 2014 AIP Publishing LLC. [http://dx.doi.org/10.1063/1.4897532]
\end{abstract}

\section{INTRODUCTION}

Mixed quantum-classical (MQC) dynamics have been a subject of interest not only in chemical physics ${ }^{1-17}$ but also in quantum gravity, ${ }^{18,19}$ cosmology, ${ }^{20,21}$ and measurement. ${ }^{22,23}$ One major problem lies in the description of correlation between the two parts, in particular, the force from the delocalized quantal part to the localized classical part, that is, the problem of "quantum backreaction." It is intimately related to the description of non-adiabatic transitions in which the Born-Oppenheimer approximation breaks down, for instance, near the conical intersections of adiabatic states. Many theories have been proposed, but the problem is normally of approximate nature. ${ }^{24-26}$ Thus, the assessment would be based not only on the theoretical consistency but also on the practical accuracy in applications. In addition, simplicity for computational implementation to realistic systems will be an important aspect.

In chemical physics, the quantum part usually represents electrons or protons, and the classical part represents heavier nuclei. For the latter, localized wave packet (WP) description, typically by Gaussian WPs, ${ }^{27,28}$ is also useful. In recent years, we have been studying a "semiquantal" (SQ) squeezedstate WP theory for chemical problems, with applications to hydrogen-bond (HB) structure and dynamics. ${ }^{29-36}$ The SQ WP takes into account the zero-point energy (ZPE) fluctuation and dynamic WP broadening, free of the ZPE leakage problem, ${ }^{37,38}$ in a canonical Hamiltonian form that realizes a stable symplectic propagation. Thus, it properly describes the WP delocalization over low-barrier HB, under-barrier shallow tunneling, and geometric isotope effects in HB structure. For instance, the critical barrier height for the adiabatic proton transfer is $\sim 2 \mathrm{kcal} / \mathrm{mol},{ }^{39}$ which hinders the classical dynamics with the thermal energy of $300 \mathrm{~K} \simeq 0.6 \mathrm{kcal} / \mathrm{mol}$, but can be properly treated by the WP delocalization. An exten-

\footnotetext{
a)E-mail: ando@kuchem.kyoto-u.ac.jp
}

sion to electron WPs with the valence-bond spin-couplings was also examined, ${ }^{40,41}$ and a combination of nuclear and electron WPs was applied to liquid hydrogen. ${ }^{42,43}$ In addition, the SQ WP can be regarded as a coherent-state basis for the path-integral formulation of quantum propagator, for which the initial value representation (IVR) has been demonstrated applicable. ${ }^{44}$ Following these, we put forward in this work a mixed quantal-semiquantal (MQSQ) theory. Related to our previous works mentioned above, the simplicity for implementations to realistic molecular systems will be the key aspect.

We start with a MQSQ trial wave function and derive the equations of motion (EOM) by the time-dependent variational principle. The resulting EOM for the SQ part have the canonical Hamiltonian form for the center and width variables of the WP. The quantal part follows a time-dependent Schrödinger equation (TDSE), in which the potential energy function is averaged over the SQ WP and thus includes the WP variables as the time-dependent external parameters. The potential function for the evolution of the SQ part is an average over both the SQ WP and quantal wave function, and thus we encounter the problem of "backreaction." To address this, we propose in this work to exploit the theory of stochastic particle (SP) dynamics. ${ }^{45-47}$ The SP dynamics are described by the stochastic differential equations (SDE) whose Fokker-Planck form is equivalent to the TDSE. We thus describe both the quantal and SQ wave functions by the corresponding sets of SPs. By assuming the pre-averaged form for the interaction between the SPs, the interparticle correlation beyond the mean-field approximation is described.

Section II describes the theory. Starting from a simple factorized form of the wave function, the mean-field (MF) limit is straightforwardly derived in Sec. II A. Then, in an aim to take into account the interparticle correlation, the SP descriptions are introduced in Sec. II B, which defines the MQSQ-SP scheme. In Sec. II C, the MQC limit is derived immediately by setting the point-particle (zero width) 
limit of the SQ WP, thereby a combination of MQC and SPs (MQC-SP) is naturally derived. Moreover, replacing the SPs by Bohmian particles is straightforward, which gives MQCB and MQSQ-B schemes. Relations to other methods and the form of the wave function are discussed in Sec. II C. Section III examines a numerical application to a model of $\mathrm{O}_{2}$ scattering from a $\mathrm{Pt}$ surface. Section IV summarizes and concludes.

\section{THEORY}

The coordinates of the quantal and SQ parts are represented by $x$ and $X$. For simplicity, we consider the SQ WP of the form ${ }^{48-50}$

$$
\begin{aligned}
\chi_{\Gamma}(X, t)= & N_{t} \exp \left[-\left(\frac{1}{4 \rho_{t}^{2}}-\frac{i}{\hbar} \frac{\Pi_{t}}{2 \rho_{t}}\right)\right. \\
& \left.\times\left(X-Q_{t}\right)^{2}+\frac{i}{\hbar} P_{t}\left(X-Q_{t}\right)\right],
\end{aligned}
$$

in which $N_{t}=1 /\left(2 \pi \rho_{t}^{2}\right)^{1 / 4}$. The WP is characterized by a set of time-dependent variables $\Gamma_{t} \equiv\left\{Q_{t}, P_{t}, \rho_{t}, \Pi_{t}\right\}$, where $Q_{t}$ and $\rho_{t}$ describe the WP center and width, $P_{t}$ and $\Pi_{t}$ are their corresponding conjugate momenta. Generalization to a correlated multi-dimensional WP, in which the variables are vectors and matrices, has been implemented for a simulation of liquid water, ${ }^{35}$ but the simpler form of Eq. (1) would be appropriate for this first presentation.

\section{A. The mean-field limit}

For the total wave function, we set forth a factorized form

$$
\psi_{\gamma \Gamma}(x, X, t)=\chi_{\Gamma}(X, t) \varphi_{\gamma \Gamma}(x, t)
$$

The idea behind this factorization will be discussed below. The subscript $\Gamma$ indicates dependence on the variables that characterize the SQ WP of Eq. (1). Similarly, $\gamma$ consists of a set of variables that characterize the quantal wave function $\varphi$; in applications to the electronic wave function, they can be the coefficients of molecular orbitals or configuration interaction, the Thouless parameters for Slater determinant, or the electron WP variables. In some cases, $\varphi$ may also depend parametrically on the SQ WP variables $\Gamma$. Recently, exact factorization of molecular wave functions to electronic and nuclear parts has been discussed. ${ }^{51,52}$ The idea here is rather simple; as we will take into account the interparticle correlation via the combination with the SP description, the factorized form Eq. (2), which naturally yields the mean-field equations as described below, should be the appropriate starting point in a sense to avoid double-counting of the correlation. (This could be considered analogous to the standard quantum chemical calculations that start with the mean-field Hartree-Fock model followed by various electron-correlation methods.) We shall come back to this matter in Sec. II C.

The time-dependence of the wave function $\psi_{\Gamma \gamma}$ is described by the variables $\Gamma_{t}$ and $\gamma_{t}$ whose EOM are derived from the time-dependent variational principle with the action integral $\mathcal{S}=\int_{t_{1}}^{t_{2}} d t\left\langle\psi(t)\left|i \hbar \partial_{t}-\hat{H}\right| \psi(t)\right\rangle$, in which

$$
\hat{H}=\hat{T}_{x}+\hat{T}_{X}+v(x, X)
$$

is the Hamiltonian with the kinetic energies $\hat{T}_{x}$ and $\hat{T}_{X}$ and the potential energy $v(x, X)$. With the trial wave function of Eq. (2), the stationary condition of the action $\mathcal{S}$ with respect to the variation of $\varphi, \delta \mathcal{S} / \delta \varphi=0$, gives

$$
i \hbar \frac{\partial}{\partial t} \varphi_{\gamma \Gamma}(x, t)=\left(\hat{T}_{x}+V\left(x ; \Gamma_{t}\right)\right) \varphi_{\gamma \Gamma}(x, t),
$$

in which $V$ is the averaged potential over the SQ WP $\chi$,

$$
V\left(x ; \Gamma_{t}\right)=\int d X\left|\chi_{\Gamma}(X, t)\right|^{2} v(x, X) .
$$

Equation (4) has a form of TDSE affected by the external time-dependent variables $\Gamma_{t}$. The variation with respect to the variables in $\chi, \delta \mathcal{S} / \delta \Gamma=0$, gives the EOM of the canonical Hamilton form

$$
\dot{Q}=\frac{\partial \tilde{H}}{\partial P}, \quad \dot{P}=-\frac{\partial \tilde{H}}{\partial Q}, \quad \dot{\rho}=\frac{\partial \tilde{H}}{\partial \Pi}, \quad \dot{\Pi}=-\frac{\partial \tilde{H}}{\partial \rho},
$$

with the Hamiltonian in the extended phase-space $\Gamma$,

$$
\tilde{H}=\frac{P^{2}}{2 M}+\frac{\Pi^{2}}{2 M}+\frac{\hbar^{2}}{8 M \rho^{2}}+U_{\gamma}(\Gamma),
$$

in which $M$ is the mass for $X$ and

$$
U_{\gamma}(\Gamma)=\int d x\left|\varphi_{\gamma \Gamma}(x)\right|^{2} V(x ; \Gamma) .
$$

\section{B. Introducing stochastic particles}

In Eq. (5), the SQ coordinate $X$ is integrated to give $V(x ; \Gamma)$, whereas in Eq. (8), both $x$ and $X$ are integrated to give $U_{\gamma}(\Gamma)$. Therefore, the dynamics of quantal and SQ parts that follow Eqs. (4)-(7) are under the mutual "mean-field," which causes the problem of describing the "backreaction." To address this, we propose in this work to deploy the theory of SP dynamics. ${ }^{45-47}$ The SP dynamics are described by the SDE,

$$
d x_{t}=\frac{\hbar}{m}\left(\nabla_{x} S+\nabla_{x} R\right) d t+\sqrt{\frac{\hbar}{m}} d W_{t},
$$

in which $m$ is the mass for $x$ and $W_{t}$ represents the standard Wiener process. The SDE for the $X$ part has the analogous form, whose specific form for the SQ WP will be presented in Eq. (12). The functions $R$ and $S$ are the real and imaginary parts of $\ln \psi(x, X, t)=R(x, X, t)+i S(x, X, t)$, and are thus related to the amplitude and the phase, respectively. The factor $\hbar \nabla_{x} S / m$ is called the "current" velocity, whereas $\hbar \nabla_{x} R / m$ is called the "osmotic" velocity. The former describes the net flow of SPs following the wave function propagation. The latter drives SPs toward the region of larger amplitude $|\psi|^{2}$. The third "stochastic" term describes the quantum diffusion of SPs. These terms will be illustrated in Eq. (12) for the case of SQ WP. The Bohmian theory employs only the current velocity, $d x / d t=\hbar \nabla S / m$, which corresponds to an assumption that the osmotic and the stochastic terms balance to cancel. 
The energy for the SP ensemble is calculated from

$$
\begin{aligned}
\langle E\rangle_{\mathrm{sp}}= & \int d x \int d X \mathrm{e}^{2 R} \\
& \times\left[\frac{\left(\hbar \nabla_{x} S\right)^{2}}{2 m}+\frac{\left(\hbar \nabla_{X} S\right)^{2}}{2 M}+V_{\mathrm{Q}}(x, X)+v(x, X)\right],
\end{aligned}
$$

in which $V_{\mathrm{Q}}$ is the "quantum potential,"

$$
V_{\mathrm{Q}}(x, X)=-\frac{\hbar^{2}}{2 m} \frac{\nabla_{x}^{2} \mathrm{e}^{R}}{\mathrm{e}^{R}}-\frac{\hbar^{2}}{2 M} \frac{\nabla_{X}^{2} \mathrm{e}^{R}}{\mathrm{e}^{R}} .
$$

Equations (10) and (11) are thus essentially equivalent to those in the Bohmian theory.

For the SQ WP of Eq. (1), the SDE is

$$
\begin{aligned}
d X_{t}= & {\left[\frac{P_{t}}{M}+\frac{\Pi_{t}}{M}\left(\frac{X_{t}-Q_{t}}{\rho_{t}}\right)-\frac{\hbar}{2 M \rho_{t}}\left(\frac{X_{t}-Q_{t}}{\rho_{t}}\right)\right] d t } \\
& +\sqrt{\frac{\hbar}{M}} d W_{t} .
\end{aligned}
$$

The first two terms in the right-hand side correspond to the current velocity originating from the phase of SQ WP. The third term is the osmotic velocity from the Gaussian amplitude of SQ WP. The first term $P / M$ represents the ordinary velocity of the WP center. The second term describes the breathing velocity of WP width, $\Pi / M$, scaled by a factor $(X-Q) / \rho$, which indicates that the particles in the regions of WP tail move faster than those near the WP center $Q$. This WP broadening is enhanced for the case of smaller WP width $\rho$. The third term is also scaled by the same factor $(X-Q) / \rho$, but has the opposite sign from the second term, which thus pulls back the SPs to the WP center. In the Bohmian theory, the osmotic and the stochastic terms that contain $\hbar$ are omitted. The integration over $X$ in Eq. (10) gives the SQ Hamiltonian $\tilde{H}$, in which $\left(P^{2}+\Pi^{2}\right) / 2 M$ results from $\left(\hbar \nabla_{X} S\right)^{2} / 2 M$ and $\hbar^{2} / 8 M \rho^{2}$ from the second term of $V_{\mathrm{Q}}$ in Eq. (11). Therefore, the quantum potential for the $X$ part is included in $\tilde{H}$. We thus find

$$
\langle E\rangle_{\mathrm{sp}}=\int d x \mathrm{e}^{2 r(x)}\left[\frac{\left(\hbar \nabla_{x} s(x)\right)^{2}}{2 m}-\frac{\hbar^{2}}{2 m} \frac{\nabla_{x}^{2} \mathrm{e}^{r(x)}}{\mathrm{e}^{r(x)}}\right]+\tilde{H},
$$

in which the functions $r(x, t)$ and $s(x, t)$ are defined by $\ln$ $\varphi(x, t)=r(x, t)+i s(x, t)$.

Equations (9) and (12) give the description equivalent to that of the guide wave function $\psi(x, X, t)$. Hence, as long as we employ the original Eqs. (4)-(8), the SPs will still be under the mutual mean-field. Now we propose to replace $V(x ; \Gamma)$ in Eq. (4) by the bare $v(x, X)$, and $U_{\gamma}(\Gamma)$ in Eq. (7) by $V(x ; \Gamma)$, in an aim to take into account the interparticle correlations. Therefore, the calculation proceeds as follows. (i) We introduce a set of SP pairs $\left\{\left(x_{\alpha}, X_{\alpha}\right)\right\}, \alpha=1,2, \cdots, N_{\text {sp }}$, distributed according to the initial probability density $|\psi(x, X, 0)|^{2}$. In practice, a random number generator can be used similarly to the Monte Carlo method. Each pair $\left(x_{\alpha}, X_{\alpha}\right)$ associates guide wave functions $\varphi_{\alpha}$ and $\chi_{\alpha}$. (ii) We propagate them by

$$
i \hbar \frac{\partial}{\partial t} \varphi_{\alpha}(x, t)=\left(\hat{T}_{x}+v\left(x, X_{\alpha}\right)\right) \varphi_{\alpha}(x, t),
$$

and Eq. (6) with

$$
\tilde{H}_{\alpha}=\frac{P_{\alpha}^{2}}{2 M}+\frac{\Pi_{\alpha}^{2}}{2 M}+\frac{\hbar^{2}}{8 M \rho_{\alpha}^{2}}+V\left(x_{\alpha} ; \Gamma_{\alpha}\right),
$$

and $\left(x_{\alpha}, X_{\alpha}\right)$ by the SDE (9) with $R$ and $S$ replaced by $r_{\alpha}$ and $s_{\alpha}$ from $\ln \varphi_{\alpha}=r_{\alpha}+i s_{\alpha}$ and the SDE (12) with the parameters $\Gamma_{\alpha}$ that describe $\chi_{\alpha}$. This scheme is denoted by MQSQ-SP. The propagation by the mean-field Eqs. (4)-(8), MQSQ-MF, conserves the total energy expectation

$$
\langle E\rangle_{\mathrm{mf}}=\langle\psi|\hat{H}| \psi\rangle=\left\langle\varphi\left|\hat{T}_{x}\right| \varphi\right\rangle+\tilde{H} .
$$

However, the conservation is lost once the SPs are introduced via Eqs. (14) and (15). In this regard, parallel investigation of these MQSQ-MF and MQSQ-SP will be useful in practical studies. Note that the loss of energy conservation in MQSQSP is solely due to the replacement of potential functions and not to the SP description: if the SPs were propagated with the guide wave functions from the MF Eqs. (4)-(8), the energy of Eq. (13), which is equivalent to Eq. (16), conserves. The energy conservation will be numerically examined in Sec. IIII.

\section{Mixed quantum-classical limit and other methods}

Before proceeding to the numerical application, we note the relation between the MQSQ-SP and the MQC schemes. By the classical point particle approximation for the heavy part,

$$
\left|\chi_{\Gamma}(X)\right|^{2} \rightarrow \delta\left(X-Q_{t}\right),
$$

we find $V\left(x ; \Gamma_{t}\right) \rightarrow v\left(x, Q_{t}\right)$ in Eq. (5), and then $U_{\gamma}(\Gamma)$ in Eq. (8) is replaced by

$$
U_{\gamma}(Q)=\int d x\left|\varphi_{\gamma Q}(x)\right|^{2} v(x, Q) .
$$

This has the form of ordinary potential energy surfaces (PES) parameterized by the heavy particles coordinates $Q$. By introducing the Bohmian particles for the quantal $x$ part and replacing the potential energy $U$ by the bare $v(x, X)$, a MQCB scheme analogous to the previous ones ${ }^{10,12}$ is obtained. We also note that the present MQSQ-SP has some similarity to the time-dependent quantum Monte Carlo method. ${ }^{53}$ The apparent and most significant difference is in the deployment of SQ WP.

Now we consider the form of the wave function and relation to other methods. A general wave function for the lightheavy systems can be written as

$$
\psi(x, X, t)=\sum_{i} \chi_{i}(X, t) \varphi_{i}(x ; X),
$$

expanded in terms of the instantaneous adiabatic or diabatic states $\varphi_{i}(x ; X)$ that contain $X$ as an external parameter rather than the dynamical variable. The standard surfacehopping method $^{3}$ replaces $\chi_{i}(X, t) \varphi_{i}(x ; X)$ by $c_{i}(t) \varphi_{i}(x ; X(t))$, where $c_{i}(t)$ is the coefficient for the $i$ th state. The heavy particle trajectories $X(t)$ are determined by the classical EOM on a PES of a single state, but with instantaneous switches (hops) among different states introduced with various rules. Some related methods based on Eq. (19) first derive coupled Schrödinger equations for the heavy part wave functions 
$\chi_{i}(X, t)$, and then take the classical limit. ${ }^{11}$ Similarly, one can derive the quantum Liouville equation for the reduced density matrix and apply the partial Wigner transformation only to the $X$ part. ${ }^{8,15}$ These methods based on Eq. (19) generally require prior knowledge of the matrix $\left\langle\varphi_{i}|\hat{H}| \varphi_{j}\right\rangle$, the adiabatic PES, and non-adiabatic couplings when the adiabatic states are employed. This causes some technical problems when continuum states are involved and the number of bound states can vary along the classical trajectories. ${ }^{7}$

Alternatively, one can start with a different factorization of the polar form

$$
\psi(x, X, t)=A(x, X, t) \exp (i S(x, X, t) / \hbar),
$$

in which $x$ and $X$ are coupled in both the amplitude and phase functions $A$ and $S$. The Bohmian theory leads to coupled EOM for $x$ and $X$ involving forces from the quantum potential $V_{\mathrm{Q}}(x, X)$ of the form Eq. (11). Various approximations are then introduced, for instance, by disregarding the quantum forces that are proportional to $1 / M^{10,12}$ or by linearizing the quantum potential to avoid its singularity problem. ${ }^{17}$ The present MQSQ-SP scheme is closer to these Bohmian approaches in that it does not require the prior construction of PES. Although the starting ansatz of Eq. (2) is apparently different, the resulting working equations include the MQCB scheme as a classical point particle limit of Eq. (17). As noted near Eq. (13), the effective Hamiltonian $\tilde{H}$ essentially includes the quantum potential for the heavy part.

\section{APPLICATION}

As a numerical demonstration, we study the same model as in Refs. 7 and 12 for gaseous $\mathrm{O}_{2}$ collision to a Pt surface, a prototype in which the ordinary MQC mean-field (MQC-MF) method fails to describe the temporal splitting of the wave function to trapped and scattered parts.

\section{A. Model}

The potential function is given by

$$
v(x, X)=\frac{1}{2} M \Omega^{2} X^{2}+a\left[\mathrm{e}^{-2 b(x-c)}-2 \mathrm{e}^{-b(x-c)}\right]+A \mathrm{e}^{-B(x-X)} .
$$

The first term is a harmonic binding potential of the heavy particle $X$ to the surface, the second term is a Morse potential for the interaction between the light particle $x$ and the surface, and the third term is a repulsive interaction between the particles. For this $v(x, X)$, the $V$ of Eq. (5) is derived as

$$
\begin{aligned}
V(x ; \Gamma)= & \frac{1}{2} M \Omega^{2}\left(Q^{2}+\rho^{2}\right)+a\left[\mathrm{e}^{-2 b(x-c)}-2 \mathrm{e}^{-b(x-c)}\right] \\
& +A \mathrm{e}^{-B(x-Q)+(B \rho)^{2} / 2} .
\end{aligned}
$$

The initial wave function at $t=0$ is set as a product of the harmonic ground-state wave function for $X$ and a Gaussian WP for $x$ centered at $x=x_{0}$ with a width $\gamma$ and the momentum $k_{0}$,

$\psi(x, X, 0)=N \exp \left[-\frac{M \Omega X^{2}}{2 \hbar}\right] \exp \left[-\frac{\left(x-x_{0}\right)^{2}}{\gamma^{2}}+\frac{i k_{0} x}{\hbar}\right]$,

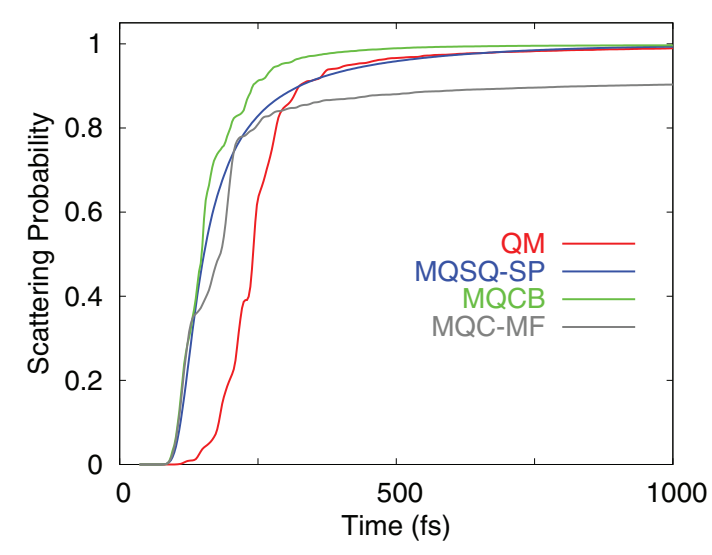

FIG. 1. Scattering probability from the methods of full quantum mechanics (QM), mixed quantal-semiquantal with stochastic particles (MQSQ$\mathrm{SP}$ ), mixed quantum-classical with Bohmian particles (MQCB), and mixed quantum-classical mean-field approximation (MQC-MF).

in which $N=\left(2 M \Omega / \pi^{2} \hbar \gamma^{2}\right)^{1 / 4}$. The initial momentum $k_{0}$ is specified by the energy $E_{0}$ via $k_{0}=-\sqrt{2 m E_{0}}$. We have taken the numerical parameters from Ref. 12: $m=1 \mathrm{amu}, M$ $=10 \mathrm{amu}, \Omega=5 \times 10^{14} \mathrm{~s}^{-1}, A=10^{4} \mathrm{~kJ} / \mathrm{mol}, B=4.25 \AA^{-1}$, $a=700 \mathrm{~kJ} / \mathrm{mol}, b=5.0 \AA^{-1}, c=0.7 \AA, x_{0}=6.0 \AA$, and $\gamma=0.5 \AA$.

The quantum mechanical (QM) wave functions were propagated using Cayley's hybrid scheme with real-space grids. ${ }^{54}$ Convergence and unitarity of the propagation were confirmed with the grid lengths $\Delta x=0.0178 \AA, \Delta X=0.0159$ $\AA$, and the time step $\Delta t=0.0124$ fs. The trajectories of ( $Q$, $P)$ and $(\rho, \Pi)$ conjugate pairs were propagated by Suzuki's symplectic fourth-order scheme. ${ }^{55}$ The transmission-free absorbing potential ${ }^{56}$ was applied to the scattered wave function along $x$. The results presented in Figs. 1-3 are with the absorbing potential set at $81 \AA<x<91 \AA$, although converged results were obtained with $45 \AA<x<51 \AA$. For the calculations in Fig. 4, it was set at $270 \AA<x<303 \AA$. For the number of SP pairs, convergence was found with $N_{\mathrm{sp}}=2000$. The same number of Bohmian particles were used in the MQCB calculation.

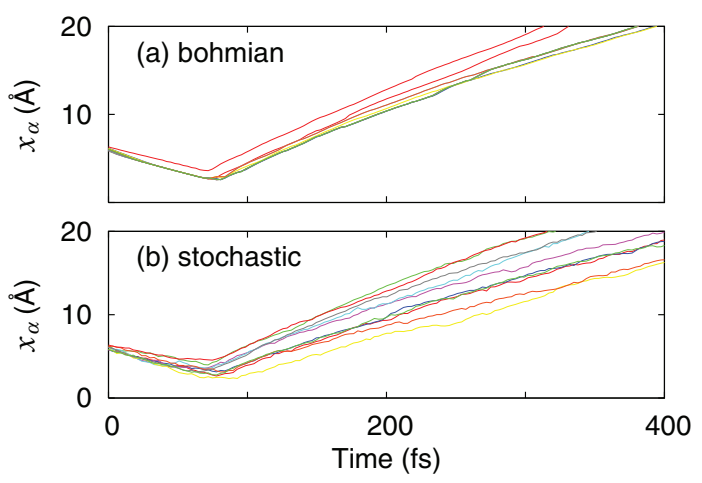

FIG. 2. Sample (ten) trajectories of (a) Bohmian particles in MQCB and (b) stochastic particles in MQSQ-SP. 


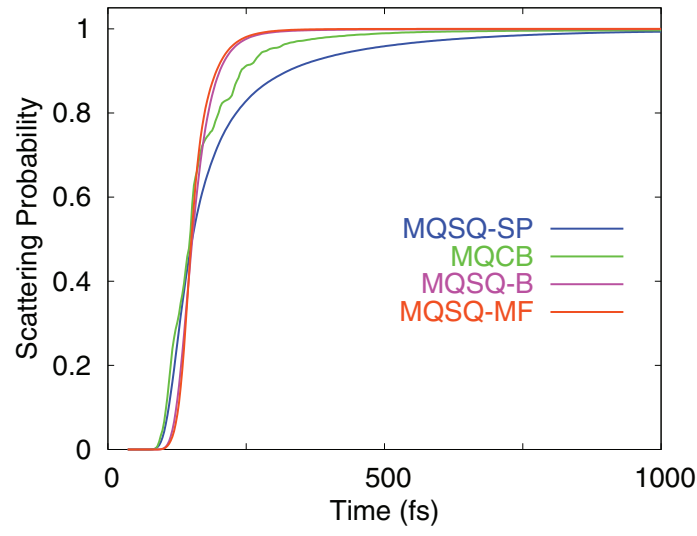

FIG. 3. Scattering probability from the methods of MQSQ-SP, MQCB, MQSQ-B, and MQSQ-MF.

\section{B. Results and discussion}

Figure 1 presents the scattering probability defined by

$$
P_{\mathrm{s}}(t)=\int_{x_{\mathrm{s}}}^{\infty} d x \int_{-\infty}^{\infty} d X|\psi(x, X, t)|^{2},
$$

with $x_{\mathrm{s}}=5.8 \AA .^{7}$ (In practice, it was calculated from the integration over $0<x<x_{\mathrm{s}}$ subtracted from unity, in order to minimize the effect of the absorbing potential.) The MQSQSP reproduces the correct asymptotic behavior, in contrast to the MQC-MF and with improvement over the MQCB. However, the description of delayed initial increase of QM $P_{\mathrm{s}}(t)$, due to the temporal resonance trapping by the heavy particle excitation, ${ }^{7}$ was still incomplete. In this regard, an intriguing further test would be to introduce dissipation to the heavy part.

In an aim to understand the improved description, we plot in Fig. 2 sample trajectories of stochastic and Bohmian particles. The difference basically emerges from the osmotic term $\hbar \nabla R / m$ and the stochastic term $\sqrt{\hbar / m} d W$ in Eq. (9); the Bohmian dynamics do not involve them but only the current velocity $d x_{t}=\hbar \nabla S / m$. This provides an understanding of the more ballistic trajectories of Bohmian particles in Fig. 2(a). However, further analysis revealed that the use of SPs alone does not account for the difference, because a combination

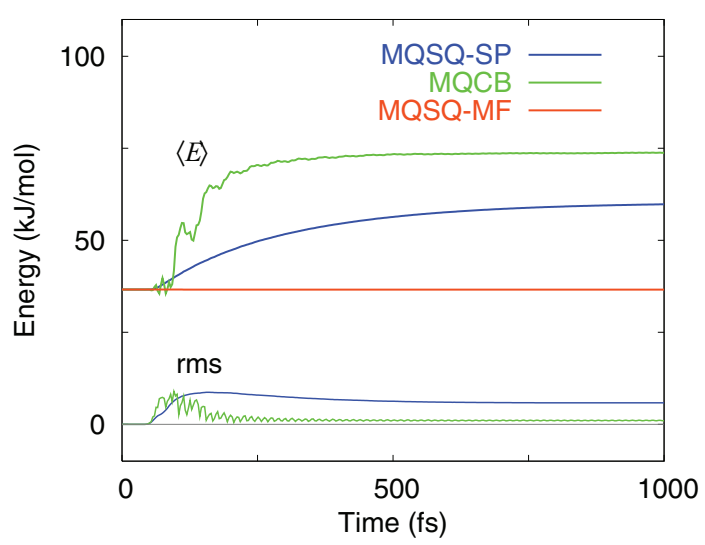

FIG. 4. Average total energies $\langle E\rangle$ for MQSQ-MF, MQSQ-SP, and MQCB from Eqs. (16), (25) and (26). For MQSQ-SP and MQCB, the root-meansquare (rms) deviations from the averages are also displayed. of MQC and SP (MQC-SP) resulted in $P_{\mathrm{s}}(t)$ almost identical to that from MQCB, which indicates that the combination of MQSQ and SP is essential for the result in Fig. 1.

To gain further insight, we compare the primary results in Fig. 1 from MQSQ-SP and MQCB with the other combinations, MQSQ-B, MQC-SP, and MQSQ-MF. Figure 3 displays the computed scattering probabilities $P_{\mathrm{s}}(t)$ from these schemes. (As noted, the result from MQC-SP was indistinguishable from that of MQCB, and thus was omitted.) The rapid increase of $P_{\mathrm{s}}(t)$ from MQSQ-MF reflects the lack of interparticle correlation. In this regard, the correlation is certainly included in MQCB and MQSQ-SP to better approximate the QM reference, as seen in Fig. 1. This aspect will be related to the results of energy conservation described next.

Figure 4 displays the numerical results of energy conservation for MQSQ-MF, MQSQ-SP, and MQCB. For MQSQ$\mathrm{MF}$, the total energy was calculated from Eq. (16). For MQSQ-SP, it was calculated from

$$
\langle E\rangle_{\mathrm{mqsq}}=N_{\mathrm{sp}}^{-1} \sum_{\alpha}\left\langle\psi_{\alpha}|\hat{H}| \psi_{\alpha}\right\rangle .
$$

The corresponding energy can be calculated also from the SP ensemble with Eq. (13). However, because we explicitly calculate the guide wave functions in this work, we employed simpler and numerically robust Eq. (25). Similarly, the energy of MQCB was calculated from

$$
\langle E\rangle_{\mathrm{mqc}}=N_{\mathrm{bp}}^{-1} \sum_{\alpha}\left(\left\langle\varphi_{\alpha}\left|\left(\hat{H}-\hat{T}_{X}\right)\right| \varphi_{\alpha}\right\rangle+\frac{P_{\alpha}^{2}}{2 M}\right),
$$

in which $N_{\mathrm{bp}}$ is the number of Bohmian trajectories. As noted in Sec. II, MQSQ-MF conserves the energy, which was numerically confirmed in the figure. For MQSQ-SP and MQCB, the energies conserve until the collision occurs at $t \simeq 100 \mathrm{fs}$. Then, they begin to increase almost monotonically toward asymptotes. The increases were up to $24 \mathrm{~kJ} / \mathrm{mol}$ for MQSQSP and $38 \mathrm{~kJ} / \mathrm{mol}$ for MQCB, which are $3 \%$ and $5 \%$ of $a=700 \mathrm{~kJ} / \mathrm{mol}$, the depth of the Morse potential for the interaction between the light particle and the surface in Eq. (21) as a measure of energy range. These extra energies could have accelerated the increase of scattering probability in comparison with the energy conserving MQSQ-MF case, but the results in Fig. 3 display the opposite, which indicates that both MQSQ-SP and MQCB effectively include the interparticle correlations, even though incompletely than the QM limit.

The increases in Fig. 4 are well-behaved without abrupt divergence or intense oscillations, and the amounts were fractional compared to the energy range involved in the process. Thus, these would be possibly controllable in ways analogous to, e.g., the momentum scaling prescription in the surface-hopping methods, although details are open to examination. The root-mean-square deviations from the averages of Eqs. (25) and (26) are also included in Fig. 4. The faster decay for MQCB is due to the ballistic nature of the trajectories as discussed with Fig. 2.

\section{CONCLUSION}

We have formulated a MQSQ theory with a SP description of the interparticle correlation, and examined it 
numerically for a prototype model involving wave function splitting. Its simplicity will be the key to implementing to realistic molecular simulations. Despite the simplicity, the results were encouraging, although a need for refining the description of interparticle correlation was still evident. We also note that the model of Eq. (21) employs for the heavy part a harmonic potential on which classical mechanics is patently appropriate. More stringent tests should clarify the nature of the present MQSQ scheme. Particularly interesting would be the cases in which the quantum mechanical aspects of the heavy part play some role, for instance, in the low-barrier HB dynamics, the ZPE leakage in dissipative systems, and others mentioned in Sec. I. Finally, as also noted in Sec. I, the IVR propagator with the SQ WP is now applicable, ${ }^{44}$ which will provide more flexible description of the wave function by the proper inclusion of quantum phase. Its integration with the present MQSQ formulation is a direction in which to proceed.

\section{ACKNOWLEDGMENTS}

The author acknowledges support from KAKENHI Nos. 22550012 and 26620007.

${ }^{1}$ P. Pechukas, Phys. Rev. 181, 166 (1969).

${ }^{2}$ H. D. Meyer and W. H. Miller, J. Chem. Phys. 70, 3214 (1979).

${ }^{3}$ J. C. Tully, J. Chem. Phys. 93, 1061 (1990).

${ }^{4}$ E. R. Bittner and P. J. Rossky, J. Chem. Phys. 103, 8130 (1995).

${ }^{5}$ D. A. Micha, Int. J. Quantum Chem. 60, 109 (1996).

${ }^{6}$ C. C. Martens and J. Y. Fang, J. Chem. Phys. 106, 4918 (1997).

${ }^{7}$ D. S. Sholl and J. C. Tully, J. Chem. Phys. 109, 7702 (1998).

${ }^{8}$ R. Kapral and G. Ciccotti, J. Chem. Phys. 110, 8919 (1999).

${ }^{9}$ M. Thoss and G. Stock, Phys. Rev. A 59, 64 (1999).

${ }^{10}$ E. Gindensperger, C. Meier, and J. A. Beswick, J. Chem. Phys. 113, 9369 (2000).

${ }^{11}$ J. C. Burant and J. C. Tully, J. Chem. Phys. 112, 6097 (2000).

${ }^{12}$ O. V. Prezhdo and C. Brooksby, Phys. Rev. Lett. 86, 3215 (2001).

${ }^{13}$ E. Deumens and Y. Öhrn, J. Phys. Chem. A 105, 2660 (2001).

${ }^{14}$ T. Terashima, M. Shiga, and S. Okazaki, J. Chem. Phys. 114, 5663 (2001).

${ }^{15}$ K. Ando and M. Santer, J. Chem. Phys. 118, 10399 (2003).

${ }^{16}$ I. Burghardt and G. Parlant, J. Chem. Phys. 120, 3055 (2004).

${ }^{17}$ S. Garashchuk and M. V. Volkov, J. Chem. Phys. 137, 074115 (2012).
${ }^{18}$ A. Anderson, Phys. Rev. Lett. 74, 621 (1995).

${ }^{19}$ J. J. Halliwell, Phys. Rev. D 57, 2337 (1998).

${ }^{20}$ B. L. Hu and S. Sinha, Phys. Rev. D 51, 1587 (1995).

${ }^{21}$ A. Campos and E. Verdaguer, Int. J. Theor. Phys. 36, 2525 (1997).

${ }^{22}$ S. Machida and M. Namiki, Prog. Theor. Phys. 63, 1833 (1980).

${ }^{23}$ W. H. Zurek, Rev. Mod. Phys. 75, 715 (2003).

${ }^{24}$ D. R. Terno, Found. Phys. 36, 102 (2006).

${ }^{25}$ L. L. Salcedo, Phys. Rev. A 85, 022127 (2012).

${ }^{26}$ C. Barceló, R. Carballo-Rublo, L. J. Garay, and R. Gómez-Escalante, Phys. Rev. A 86, 042120 (2012).

${ }^{27}$ E. J. Heller, J. Chem. Phys. 64, 63 (1976).

${ }^{28}$ R. G. Littlejohn, Phys. Rep. 138, 193 (1986).

${ }^{29}$ K. Ando, J. Chem. Phys. 121, 7136 (2004).

${ }^{30}$ K. Ando, Phys. Rev. B 72, 172104 (2005).

${ }^{31}$ K. Ando, J. Chem. Phys. 125, 014104 (2006).

${ }^{32}$ N. Sakumichi and K. Ando, J. Chem. Phys. 128, 164516 (2008).

${ }^{33}$ K. Hyeon-Deuk and K. Ando, J. Chem. Phys. 131, 064501 (2009).

${ }^{34}$ K. Hyeon-Deuk and K. Ando, J. Chem. Phys. 132, 164507 (2010).

${ }^{35}$ J. Ono and K. Ando, J. Chem. Phys. 137, 174503 (2012).

${ }^{36}$ J. Ono, K. Hyeon-Deuk, and K. Ando, Int. J. Quantum Chem. 113, 356 (2013).

${ }^{37}$ S. D. Ivanov, A. Witt, M. Shiga, and D. Marx, J. Chem. Phys. 132, 031101 (2010).

${ }^{38}$ S. Habershon, D. E. Manolopoulos, T. E. Markland, and T. F. Miller III, Annu. Rev. Phys. Chem. 64, 387 (2013).

${ }^{39}$ K. Ando and J. T. Hynes, Adv. Chem. Phys. 110, 381 (1999).

${ }^{40}$ K. Ando, Bull. Chem. Soc. Jpn. 82, 975 (2009).

${ }^{41}$ K. Ando, Chem. Phys. Lett. 523, 134 (2012).

${ }^{42}$ K. Hyeon-Deuk and K. Ando, Chem. Phys. Lett. 532, 124 (2012).

${ }^{43}$ K. Hyeon-Deuk and K. Ando, J. Chem. Phys. 140, 171101 (2014).

${ }^{44}$ K. Ando, Chem. Phys. Lett. 591, 179 (2014).

${ }^{45}$ E. Nelson, Phys. Rev. 150, 1079 (1966).

${ }^{46}$ K. Yasue, J. Funct. Anal. 41, 327 (1981).

${ }^{47}$ K. Yasue and J. C. Zambrini, Ann. Phys. 159, 99 (1985).

${ }^{48}$ F. Arickx, J. Broeckhove, E. Kesteloot, L. Lathouwers, and P. van Leuven, Chem. Phys. Lett. 128, 310 (1986).

${ }^{49}$ Y. Tsue, Prog. Theor. Phys. 88, 911 (1992).

${ }^{50}$ A. K. Pattanayak and W. C. Schieve, Phys. Rev. E 50, 3601 (1994).

${ }^{51}$ A. Abedi, N. T. Maitra, and E. K. U. Gross, Phys. Rev. Lett. 105, 123002 (2010).

${ }^{52}$ L. S. Cederbaum, J. Chem. Phys. 138, 224110 (2013).

${ }^{53}$ I. P. Christov, J. Chem. Phys. 127, 134110 (2007).

${ }^{54}$ N. Watanabe and M. Tsukada, Phys. Rev. E 62, 2914 (2000).

${ }^{55}$ M. Suzuki, Phys. Lett. A 146, 319 (1990).

${ }^{56}$ T. Gonzalez-Lezana, E. J. Rackham, and D. E. Manolopoulos, J. Chem. Phys. 120, 2247 (2004). 